\title{
EchoGéo
}

$14 \mid 2010$

Afrique, 50 ans d'indépendance : Dynamiques spatiales, identités, circulations

\section{Modification des conditions de maturation du raisin en Bourgogne viticole liée au réchauffement climatique}

L'exemple des vignobles de la Côte et des Hautes-Côtes de Beaune

Malika Madelin, Benjamin Bois et Jean-Pierre Chabin

\section{OpenEdition}

Journals

Édition électronique

URL : https://journals.openedition.org/echogeo/12176

DOI : 10.4000/echogeo.12176

ISSN : 1963-1197

Éditeur

Pôle de recherche pour l'organisation et la diffusion de l'information géographique (CNRS UMR 8586)

Référence électronique

Malika Madelin, Benjamin Bois et Jean-Pierre Chabin, « Modification des conditions de maturation du raisin en Bourgogne viticole liée au réchauffement climatique », EchoGéo [En ligne], 14 | 2010, mis en ligne le 13 décembre 2010, consulté le 31 juillet 2021. URL : http://journals.openedition.org/echogeo/ 12176 ; DOI : https://doi.org/10.4000/echogeo.12176

Ce document a été généré automatiquement le 31 juillet 2021.

EchoGéo est mis à disposition selon les termes de la licence Creative Commons Attribution - Pas d'Utilisation Commerciale - Pas de Modification 4.0 International (CC BY-NC-ND) 


\section{Modification des conditions de maturation du raisin en Bourgogne viticole liée au réchauffement climatique}

L'exemple des vignobles de la Côte et des Hautes-Côtes de Beaune

Malika Madelin, Benjamin Bois et Jean-Pierre Chabin

Les auteurs remercient M. Pouleau, observateur météorologique et agriculteur à La Rochepot, $M$. Thévenin et toute l'équipe du Centre Départemental de la Météorologie de la Côte-d'Or pour l'accès aux données et les multiples informations complémentaires sur les sites de mesures, $M$. Magnien et M. Boyer du Service régional de la protection des végétaux à Beaune, qui nous ont permis d'actualiser le fichier des dates phénologiques de Beaune, Mmes Meurgues et Monamy du $B I V B$ pour leurs informations techniques.

1 Une des spécificités de la viticulture de qualité est son étroite dépendance au climat, ses fluctuations interannuelles influençant le rendement et les caractéristiques de la vendange. Les vignobles bourguignons actuels, résultats d'un empirisme séculaire, ont conduit à une spécialisation des productions régionales en relation avec les aptitudes climatiques du milieu (adaptation de l'encépagement, des pratiques viticoles et œnologiques). La question du changement climatique et de ses répercussions sur la viticulture intéresse fortement la profession viticole : quelles nouvelles géographies des aires viticoles potentielles se dessinent? Les risques phytosanitaires ou climatiques vont-ils diminuer ou augmenter? Ou encore, quelles seront les pratiques culturales à adopter pour s'adapter? Ces interrogations ont suscité de nombreuses recherches, à différentes échelles, pour plusieurs vignobles (Duchêne et Schneider, 2005 ; Jones et al., 2005 ; Kenny et Harrisson, 1992 ; Schultz, 2000, Seguin \& Garcia de Cortazar Atauri, 2005). A partir d'observations, ces études montrent des impacts convergents du changement climatique sur la viticulture, principalement liés à la hausse des températures : précocité des stades de développement, modifications des rendements et de la production foliaire, etc. S'il s'avère que les relations climat-vigne ont déjà été 
étudiées en Bourgogne (Gadille, 1967 ; Bonnardot, 1996 ; Chabin, 2004), cette question très médiatisée $d u$ changement climatique n'y a été abordée que assez récemment (Chabin et al., 2007; Madelin et al., 2008). Dans cet article, nous nous proposons d'exposer succinctement le réchauffement climatique depuis 1950 sur la région Bourgogne et son influence sur la vigne, à partir d'indices bioclimatiques fréquemment utilisés en viticulture. Une approche comparative plus fine sur deux sites du Beaunois, un sur la Côte à basse altitude (Savigny-lès-Beaune) et un sur un site d'altitude en Hautes-Côtes (La Rochepot) permettra de préciser les effets du réchauffement.

\section{Les vignobles de Bourgogne}

Les vignobles de Bourgogne, situés dans le nord-est de la France, s'étendent sur près de 30000 ha, pour une production d'environ 1,5 million d'hectolitres (BIVB, 2010). Leur répartition (figure 1), certes assez morcelée dans l'espace, s'organise en deux ensembles, de part et d'autre du seuil de Bourgogne (ligne de partage des eaux entre le bassin de la Seine et celui de la Saône, orientée NE-SW) : à l'est, le principal ensemble, situé sur la bordure occidentale de la plaine de Saône, de Dijon à la limite méridionale de la région au sud de Mâcon; au nord-ouest, dans l'Yonne et la Nièvre, où s'étendent des vignobles circonscrits aux vallées du sud du Bassin parisien: Armançon (Tonnerrois), Serein (Chablisien), Yonne (Auxerrois) et Loire (Sancerrois). Ainsi, l'interprofession viticole individualise cinq grandes régions: les vignobles de Chablis, du Grand Auxerrois, de Tonnerre, de Joigny et de Vézelay ; les vignobles de la Côte de Nuits, Hautes-Côtes de Nuits et du Châtillonnais; les vignobles de la Côte de Beaune et des Hautes-Côtes de Beaune ; les vignobles de la Côte Chalonnaise et du Couchois et enfin, le vignoble du Mâconnais (BIVB, 2010). 
Figure 1 - Relief de la Bourgogne et localisation des vignobles

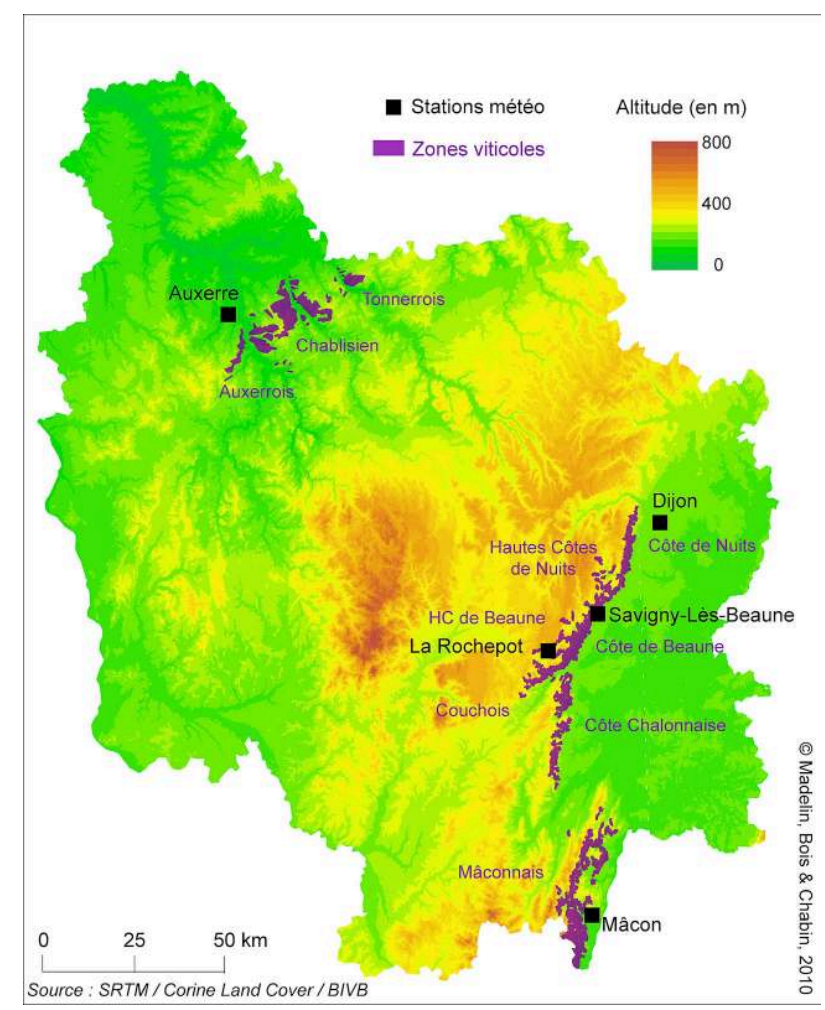

Source : SRTM/Corine Land Cover/BIVB ; auteurs : Madelin, Bois et Chabin, 2010

3 Le climat régional de la Bourgogne est globalement défini comme étant de type océanique à tendance continentale. Bonnardot (1996) a mis en évidence les conditions particulières des climats viticoles dans la Bourgogne orientale, qui présente une bonne exposition au soleil liée à l'orientation des coteaux principalement à l'est et une tendance chaude et sèche durant la période végétative, tout en évitant les contraintes du climat méditerranéen en termes de stress hydrique. Ces avantages climatiques expliquent en partie la localisation des vignobles en Bourgogne (Chabin, 2004). À l'intérieur des cinq principales régions, des distinctions qualitatives et hiérarchiques sont faites à une échelle plus fine, topoclimatique, en fonction principalement de l'altitude (Côtes / Hautes-Côtes), de la position sur les coteaux et des conditions édaphiques.

\section{Les données utilisées}

4 Pour mettre en évidence le réchauffement climatique en Bourgogne, nous avons retenu les données thermiques de stations "de référence ", telles que celles issues des mesures des stations météorologiques du réseau synoptique de Météo-France, qui permettent de fixer le cadre régional et son évolution. Situées à proximité des vignobles, elles présentent des séries climatiques homogènes et avec une relative profondeur temporelle : ici, les stations de Dijon ${ }^{1}$, d'Auxerre et de Mâcon-Charnay (série 1951-2010; figure 1). À une échelle plus fine, nous avons choisi deux stations du Beaunois implantées dans l'environnement immédiat des vignobles, pour lesquelles nous disposons de données depuis une trentaine d'années (série 1973-2010): Savigny-lèsBeaune à $220 \mathrm{~m}$ en Côte de Beaune et La Rochepot à $410 \mathrm{~m}$ dans les Hautes-Côtes de 
Beaune. Pour toutes les stations, les variables climatiques retenues sont les températures minimales et maximales journalières et mensuelles sur la période d'avril à septembre, classiquement considérée comme la «période phénologique » (de la reprise de la croissance végétative aux vendanges).

\section{Un réchauffement régional du climat, marqué depuis la fin des années 1980}

5 Les figures 2 (pour les stations « régionales ») et 3 (pour les deux stations du Beaunois) représentent l'évolution en années successives des températures moyennes de la période phénologique, ainsi que leurs moyennes mobiles sur cinq ans. Outre la forte variabilité interannuelle, ces courbes montrent, pour les séries longues, deux périodes qui se succèdent, corroborant l'évolution thermique générale en France : plateau des moyennes thermiques au cours des années 1950 à 1970, puis remontée sensible et durable à partir des années 1980. Presque toutes les séries étudiées présentent une rupture significative en 1987-1988 (tests de Pettitt, de Lee, etc.), qui est donc caractéristique d'une rupture thermique, observée dans d'autres régions viticoles (Briche et al., 2010) et en France en général (Moisselin et al., 2002). Les différences observées entre les stations témoignent d'un gradient latitudinal (Dijon / Mâcon), continental (Auxerre / Dijon) et altitudinal (Côte / Hautes-Côtes) qui explique en partie la différenciation qualitative des appellations. Les répercussions sur la viticulture de ce réchauffement climatique s'observent sur l'augmentation des rendements et sur la précocité des dates phénologiques et des vendanges (Chabin et al., 2007 ; Madelin et al., 2008), même si d'autres facteurs entrent en jeu : ainsi, les vendanges avaient lieu fin septembre dans les années 1960-1970 en Côte de Beaune, alors qu'elles y ont été avancées à la mi-septembre à la fin du $20^{\mathrm{e}}$ siècle et au début du $21^{\mathrm{e}}$ siècle. 
Figure 2 - Évolution des températures moyennes d'avril à septembre pour trois stations régionales de Bourgogne

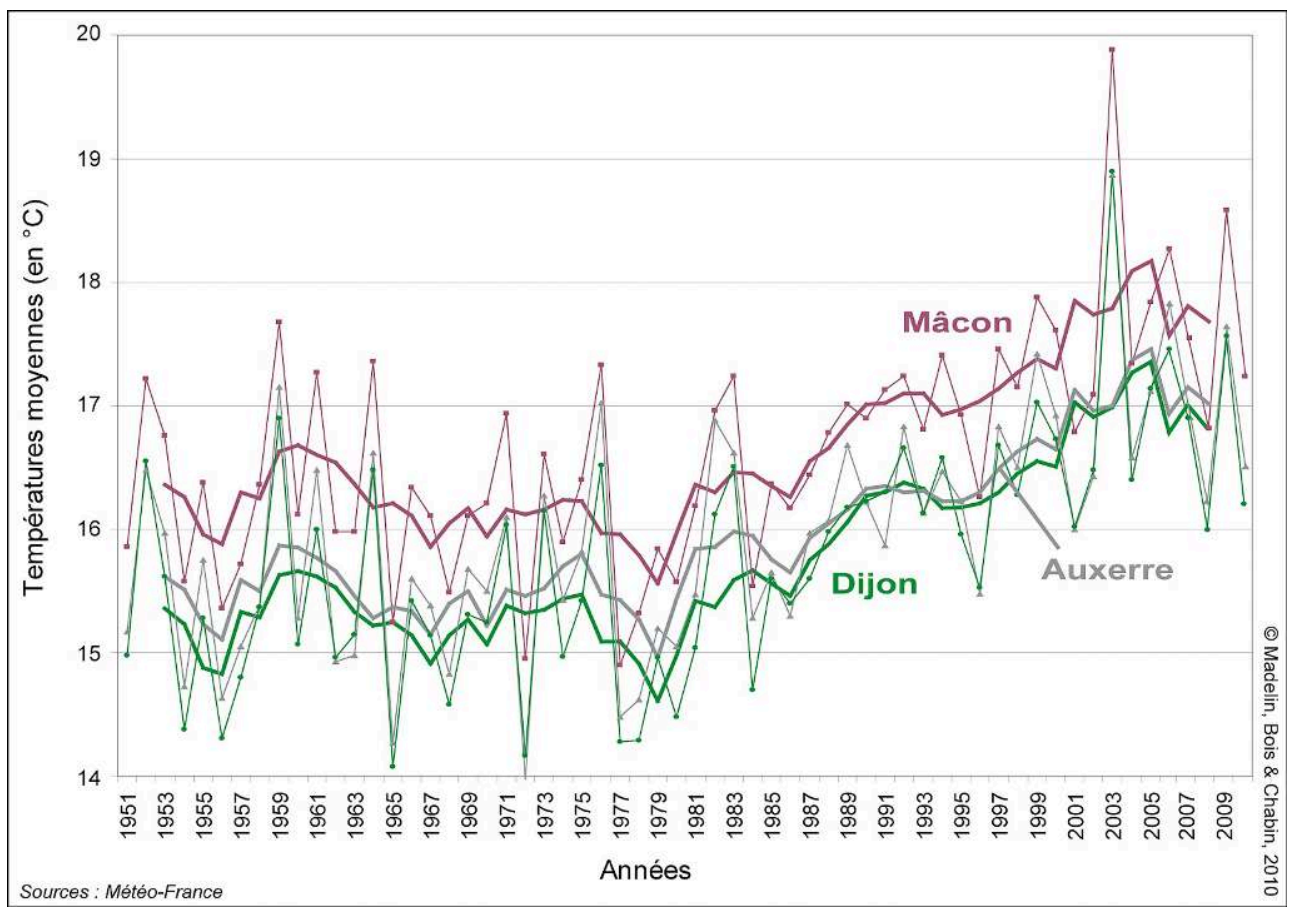

Courbes en trait épais : lissage sur 5 ans.

Source : Météo-France ; auteurs : Madelin, Bois et Chabin, 2010. 
Figure 3 : Évolution des températures moyennes d'avril à septembre pour deux stations implantées à proximité immédiate des vignobles

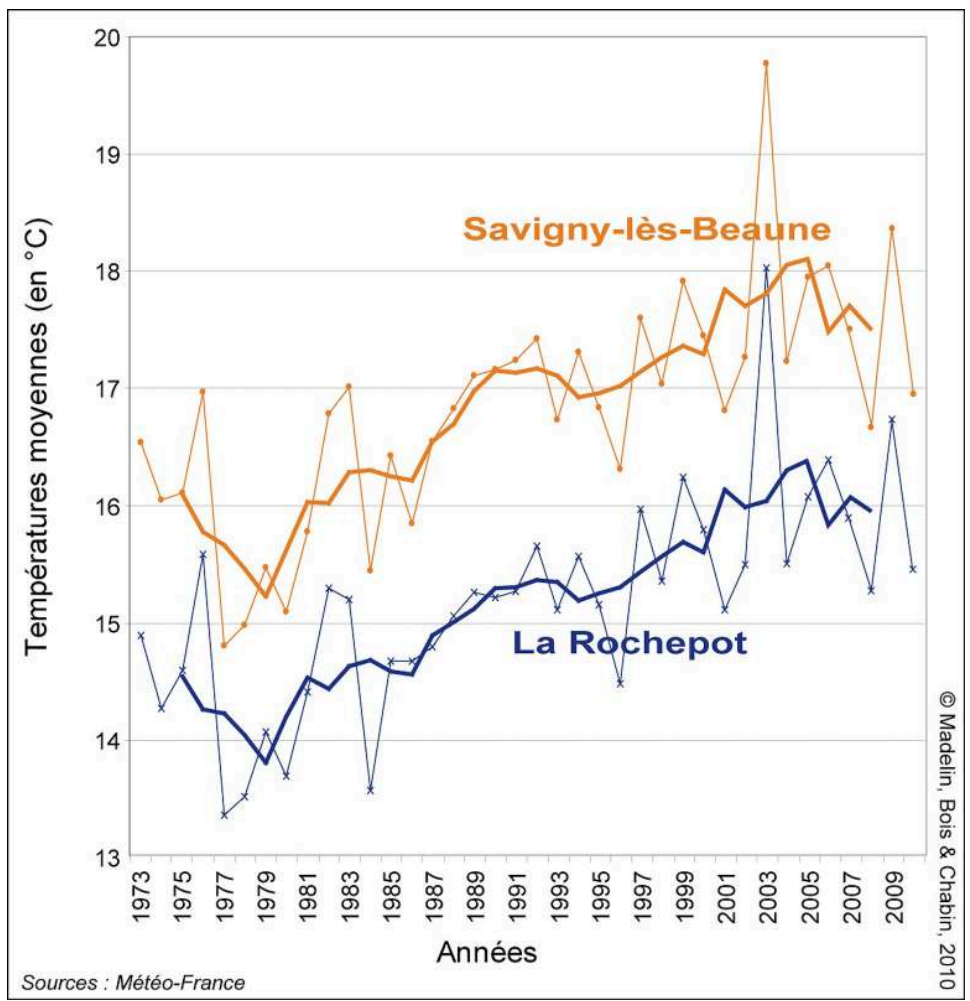

Courbes en trait épais : lissage sur 5 ans

Source : Météo-France ; auteurs : Madelin, Bois et Chabin, 2010.

\section{Les indices viticoles témoignant de conditions thermiques plus chaudes}

Nous avons ensuite calculé un indicateur classiquement mis en œuvre dans les études phénologiques, les degrés-jours, qui sont la somme des températures journalières supérieures au zéro de végétation, dont la valeur de $10^{\circ} \mathrm{C}$ est globalement admise pour la vigne (Amerine et Winkler, 1944; Huglin, 1978). La figure 4 représente, pour les stations de Savigny-Lès-Beaune et de La Rochepot, l'évolution temporelle de ces degrésjours sur deux périodes : avant la rupture statistique (1973-1987) et après (1988-2010). Outre le décalage entre les deux stations, d'environ 260 degrés-jours en fin de période, lié à la différence d'altitude de près de $200 \mathrm{~m}$, nous remarquons que les degrés-jours pour la période récente de La Rochepot correspondent aux conditions thermiques de Savigny-Lès-Beaune, il y a 30 ans. 
Figure 4 - Evolution de la somme des degrés-jours (du 1er avril au 30 septembre) pour les stations de Savigny-Lès-Beaune et de La Rochepot et repérage temporel des stades de développement (d'après Madelin et al., 2008)

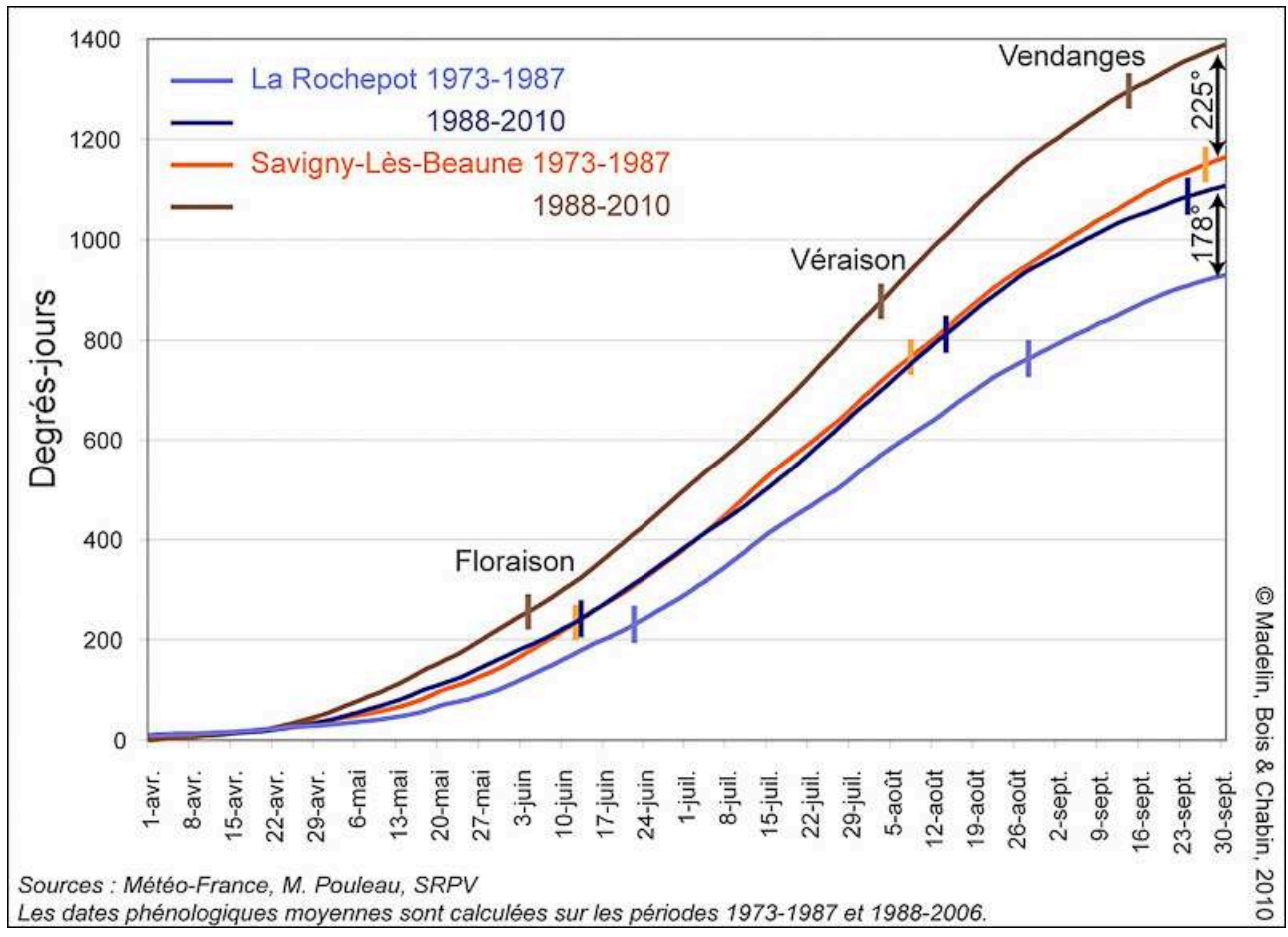

Sources : Météo-France, M.Pouleau, SRPV ; auteurs : Madelin, Bois et Chabin, 2010.

Pour ces deux stations, nous avons ensuite calculé l'indice héliothermique de Huglin (1978), très souvent utilisé en viticulture pour définir les «climats viticoles » et les aptitudes viticoles d'une région d'un point de vue climatique :

$\sum_{01 / 04}^{30 / 09} \frac{\left(T_{m}-10\right)+\left(T_{x}-10\right)}{2} \cdot K$

Pour les jours où la température moyenne est supérieure à $10^{\circ} \mathrm{C}$ :

avec $\mathrm{Tm}$ la température moyenne journalière $\left(\mathrm{en}{ }^{\circ} \mathrm{C}\right)$,

Tx la température maximale journalière $\left(\mathrm{en}{ }^{\circ} \mathrm{C}\right)$

et $\mathrm{K}$ le coefficient de longueur du jour (variant de 1,02 à 1,06 entre 40 et $50^{\circ}$ de latitude).

Depuis 1973, les conditions de La Rochepot sont ainsi passées, en regard de la classification climatique multicritère géoviticole proposée par Tonietto et Carbonneau (2004), d'un climat dit très frais à un climat frais (figure 5), tandis que celles de SavignyLès-Beaune sont de plus en plus souvent tempérées depuis la fin des années 1980. Ainsi, ces stations situées à $47^{\circ}$ de latitude nord, ont acquis en 20 ans des températures caractéristiques de régions plus basses en altitude pour La Rochepot et plus méridionales pour Savigny-lès-Beaune. Quant à l'année 2003, exceptionnellement chaude, elle place Savigny-lès-Beaune sous les conditions thermiques du Midi méditerranéen français. Des étés comme celui-là devraient devenir plus fréquents dans les décennies à venir (IPCC, 2007). 
Figure 5 - Evolution des indices de Huglin à Savigny-lès-Beaune et La Rochepot, depuis 1973

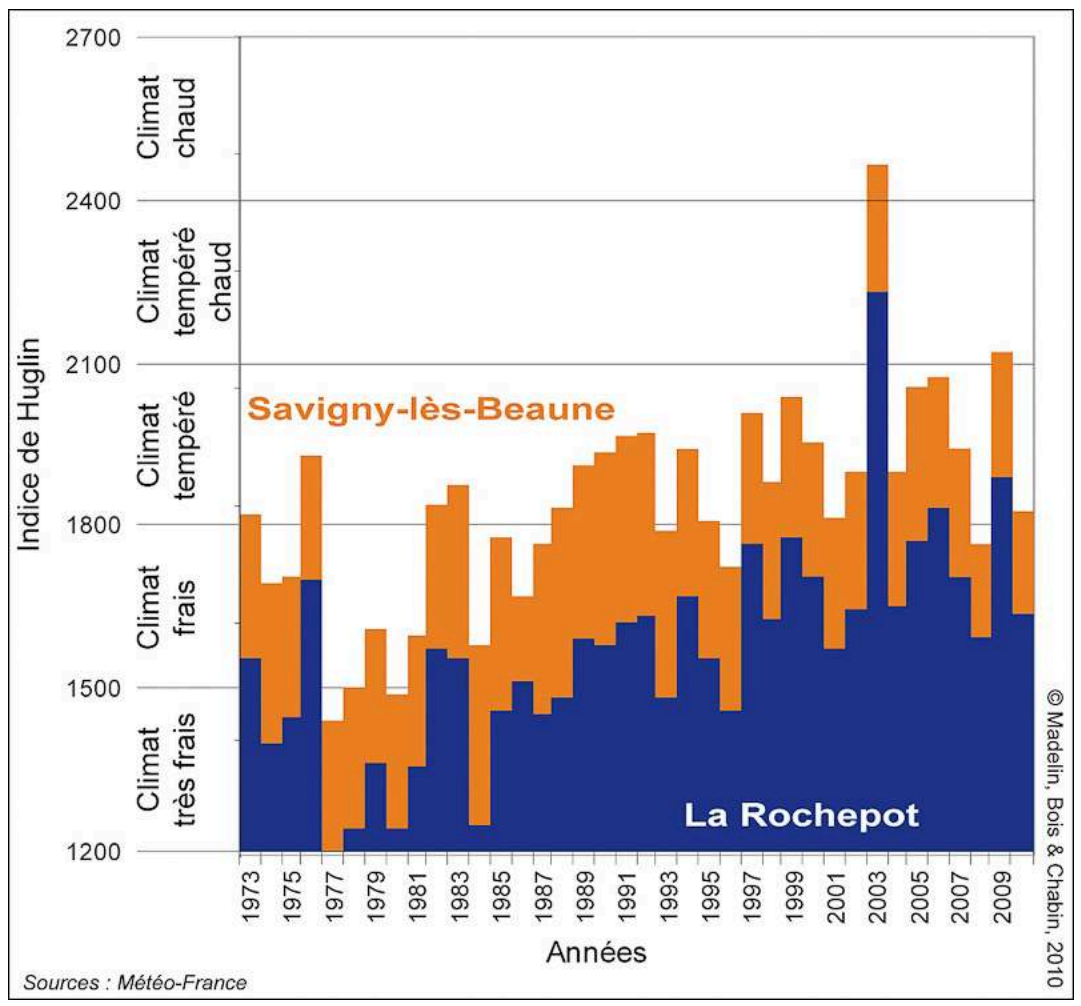

Source : Météo-France ; auteurs : Madelin, Bois et Chabin, 2010.

\section{Vers une profonde modification des conditions nycthermiques pendant la maturation}

La maturation, entre véraison et maturité-vendanges, est la période essentielle pour la qualité future de la récolte (Bonnardot, 1996), par la synthèse des composés organiques influençant la typicité du vin (sucres, acidité, polyphénols, précurseurs d'arômes) et sa signature terroir-millésime (Van Leeuwen et al., 2004). En particulier, l'effet négatif des températures minimales élevées en période de maturation du raisin est souvent souligné dans la littérature (Kliewer, 1973; Mori et al., 2005) : l'indice de Fraîcheur des nuits, défini comme la moyenne des minima thermiques durant le mois de septembre pour l'hémisphère nord (Tonietto et Carbonneau, 2004), permet ainsi d'évaluer les conditions nycthermiques pendant la maturation.

Des travaux antérieurs (Duchêne et Schneider, 2005 ; Chabin et al., 2007) ont montré une précocité plus nette vers la maturité et donc un raccourcissement progressif et significatif de la période entre la reprise de la croissance végétative (débourrement) et la date des vendanges. Par conséquent, les vignobles connaissent un « double effet » du réchauffement climatique lors de la maturation : à l'effet propre de l'augmentation des températures, se surimpose celui lié à l'avancée sur le mois d'août de la période de maturation. La maturation se fait désormais plus vite et plus brutalement qu'autrefois. La figure 6 illustre cette accentuation pour la station de Savigny-lès-Beaune, pour laquelle nous disposons de relevés phénologiques ${ }^{2}$ à proximité (stades de développement du Pinot noir relevés à Beaune par le Service Régional de la Protection 
des Végétaux): les températures moyennes minimales sur une période de 35 jours $^{3}$ avant les vendanges augmentent significativement plus rapidement que celles de septembre, en d'autres termes que l'indice de Fraîcheur des Nuits (Tonietto et Carbonneau, 2004). Les pentes des régressions réalisées (sans 2003 qui présente des conditions très particulières) montrent un rapport du simple au double. Ces nouvelles conditions lors de la maturation pourront modifier dans le futur les vins produits. Enfin, soulignons qu'au-delà du réchauffement climatique mis en évidence ici, Thévenin (2007) montre aussi une tendance à la sécheresse (à partir de l'humidité relative et de l'évapotranspiration potentielle) pendant le mois d'août à Dijon, depuis le début des années 1990.

Figure 6 - Evolution de l'indice de Fraîcheur des Nuits et de la moyenne des températures minimales sur les 35 jours précédant les vendanges pour la station de Savigny-lès-Beaune

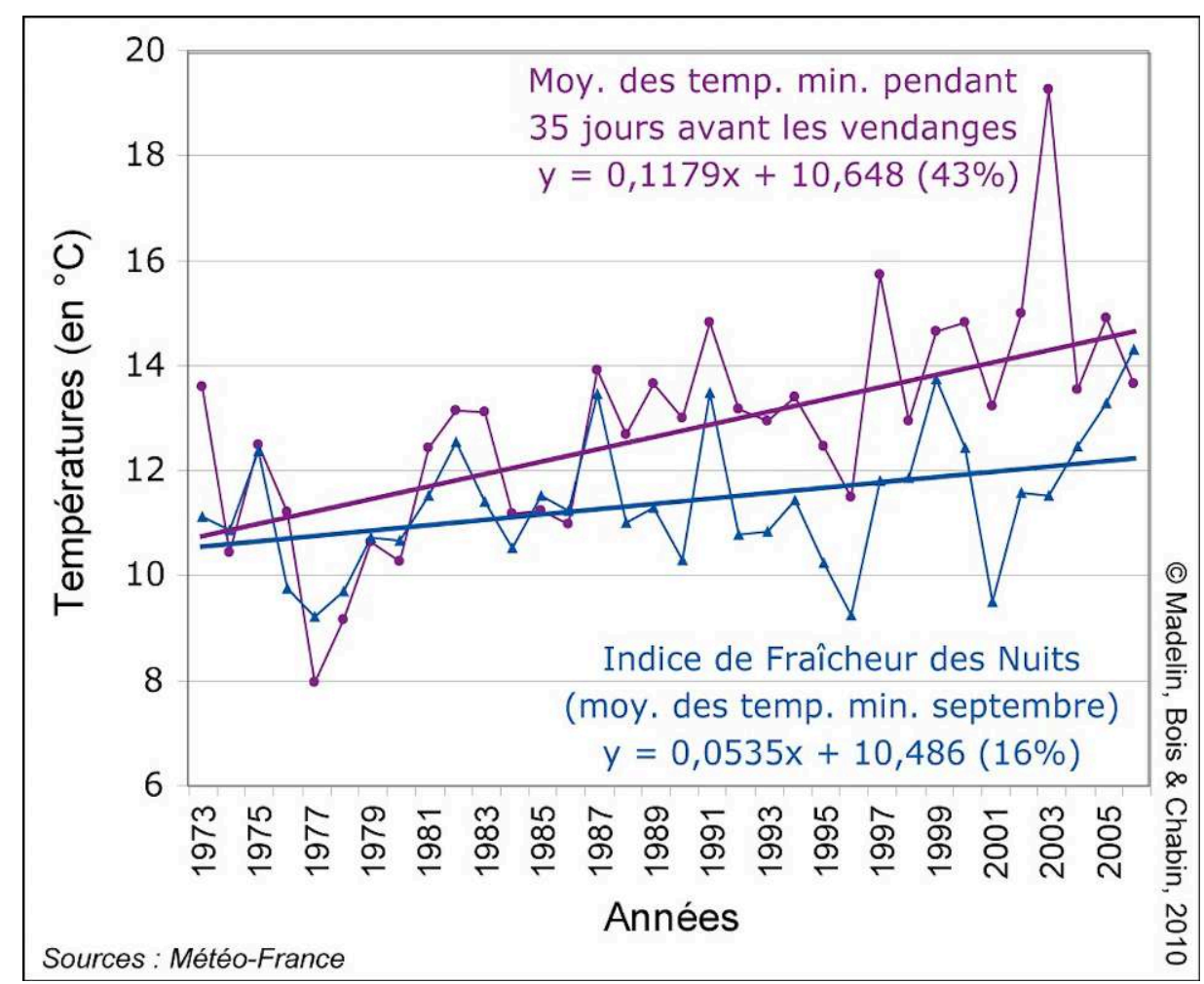

Source : Météo-France ; auteurs : Madelin, Bois et Chabin, 2010.

\section{Conclusion}

Dans le contexte actuel des bouleversements de la planète viticole et de l'intensification des concurrences, les viticulteurs des pays traditionnels se défendent en mettant en valeur le terroir, résultant des interactions entre des facteurs humains, édaphiques et climatiques. Cette notion est particulièrement mise en avant en Bourgogne pour valoriser la typicité et l'originalité des vins : ne compte-t-on pas quelque 1200 terroirs différents rien que sur la Côte d'Or ?Le réchauffement climatique mis en évidence ici traduit une évolution rapide du milieu naturel des terroirs. Le prolongement de cette recherche sera d'estimer les conséquences multiples de ce changement climatique à l'échelle de la Bourgogne, ce qui permettra à plus long terme de mieux estimer les 
conséquences économiques et sociales probables et d'orienter les adaptations des pratiques culturales.

\section{BIBLIOGRAPHIE}

Amerine M.A. \& Winkler A.J., 1944. Composition and quality of musts and wines of California grapes. Hilgardia, 15, p. 493-673.

BIVB, 2010. Site internet consulté en avril 2010 : www.vins-bourgogne.fr

Bonnardot V., 1996. Le climat et la vigne en Bourgogne Orientale. Thèse de Doctorat de Géographie, Université de Bourgogne, Dijon.

Briche E., Madelin M., Beltrando G. \& Kergomard C., 2010. Analyse comparative des températures extrêmes de 1950 à 2100 issues des modèles ARPEGE-Climat et LMD : intérêt pour l'activité viticole champenoise. La Climatologie, à paraître.

Chabin J.-P., Madelin M. \& Bonnefoy C., 2007. Les vignobles beaunois face au réchauffement climatique. Actes du colloque international Réchauffement climatique, quels impacts probables sur les vignobles ?, Dijon, France, 13 p. http://www.u-bourgogne.fr/chairevinetculture/Actes \%20clima/ Actes/Article_Pdf/Chabin.pdf

Chabin J.-P., 2004. L'excellence aux limites... ou le paradoxe des vignobles septentrionaux français d'après l'exemple côte-d'orien. Revue géographique de l'Est, 44, 1-2, p. 9-16.

Duchêne E. \& Schneider C., 2005. Grapevine and climatic changes : a glance at the situation in Alsace. Agronomy for Sustainable Development, 25, p. 93-99.

Gadille R., 1967. Le vignoble de la Côte bourguignonne : fondements physiques et humains d'une viticulture de haute qualité. Les Belles Lettres, Paris, 686 p.

Huglin P., 1978. Nouveau mode d'évaluation des possibilités héliothermiques d'un milieu viticole. Comptes Rendus de l'Académie d'Agriculture, 64, 13, p. 1117-1126.

IPCC, 2007. Rapport du groupe de travail I du GIEC : Bilan 2007 des changements climatiques : les bases scientifiques physiques. http://www.ipcc.ch/

Jones G.V., White M.A., Cooper O.R. \& Storchmann K., 2005. Climate change and global wine quality. Climatic Change, 73, 3, p. 319-343. http://www.springerlink.com/index/ PX5H048746456M63.pdf

Kenny G.J. \& Harrison P.A., 1992. The effects of climate variability and change on grape suitability in Europe. Journal of Wine Research, 3, 3, p. 163-183. http://www.informaworld.com/index/ 773493659.pdf

Kliewer W.M., 1973. Berry composition of Vitis vinifera cultivars as influenced by photo and nyctotemperatures during maturation. Journal of the American Society for Horticultural Science, 2, p. 153-159.

Madelin M., Chabin J.-P. \& Bonnefoy C., 2008. Global warming and its consequences on the Beaune vineyards. Enometrica, 1, 2, p. 9-19. 
Moisselin J-M., Schneider M., Canellas C. \& Mestre O., 2002. Les changements climatiques en France au XXème siècle. Etude des longues séries de données homogénéisées françaises de précipitations et températures. La Météorologie, 38, p. 45-46.

Mori K., Saito H., Goto-Yamamoto N., Kobayashi S., Sugaya S., Gemma H. \& Hashizume K., 2005. Effects of abscisic acid treatment and night temperatures on anthocyanin composition in Pinot noir grapes. Vitis, 44, p. 161-165.

Schultz H.R., 2000. Climate change and viticulture: a European perspective on climatology, carbon dioxide, and UV-B effects. Australian Journal of Grape and Wine Research, 6, p. 2-12. http:// www3.interscience.wiley.com/journal/120138640/articletext?DOI=10.1111\%2Fj. 1755-0238.2000.tb00156.x

Seguin B. \& Garcia de Cortazar Atauri I., 2005. Climate warming: consequences for viticulture and the notion of terroirs in Europe. Acta Horticulturae, 689, p. 61-71. http://www.actahort.org/ books/689/689_3.htm

Thevenin D., 2007. Diminution de l'humidité avant maturité en Bourgogne à la fin du XX $\mathrm{X}^{\mathrm{e}}$ siècle. Poster lors du colloque international Réchauffement climatique, quels impacts probables sur les vignobles?, Dijon, France.

Tonietto J. \& Carbonneau A., 2004. A multicriteria climatic classification system for grapegrowing regions worldwide. Agricultural and Forest Meteorology, 124, 1-2, p. 81-97. http:// linkinghub.elsevier.com/retrieve/pii/S0168192304000115

Van Leeuwen C., Friant P., Choné X., Tregoat O., Koundouras S. \& Dubourdieu D., 2004. Influence of climate, soil, and cultivar on terroir. American Journal of Enology and Viticulture, 55, 3, p. 207-217. http://www.ajevonline.org/cgi/content/abstract/55/3/207

\section{NOTES}

1. Notons le changement de site, en 1982, de Dijon-Ouges à Dijon-Longvic: les données antérieures à cette date et utilisées ici sont celles réajustées par Météo-France à l'échelle mensuelle (communication personnelle du CDM de la Côte-d'Or, d'après Moisselin et al., 2002).

2. Seulement de 1973 à 2006.

3. Le seuil de 35 jours choisi ici correspond à la période la plus courte, depuis 1973, entre véraison et vendanges à Beaune (qui a eu lieu en 2003). Ici, la moyenne des températures minimales sur 35 jours avant les vendanges est comparable à l'indice de Fraîcheur des nuits (calculé sur les jours de septembre).

\section{RÉSUMÉS}

Les répercussions du changement climatique, en particulier celles qui sont liées à l'augmentation des températures, s'observent dans de nombreux vignobles dans le monde. En Bourgogne, depuis 20 ans, cette évolution thermique modifie les conditions de la maturation, moment-clef pour la typicité du vin et sa signature terroir-millésime. Après une étude de l'évolution des températures en Bourgogne montrant en particulier une rupture en 1987-1988, une analyse comparée des 
conséquences phénologiques, à partir des données thermiques et des dates des stades de développement, est proposée pour deux sites côte-d'oriens en Côte et en Hautes-Côtes de Beaune (Savigny-lès-Beaune et La Rochepot). Les résultats montrent un transfert latitudinal et altitudinal des températures et des indices viticoles sur 20-30 ans, ainsi qu'un «double effet »du réchauffement climatique lors de la maturation, lié à l'augmentation des températures et au décalage temporel de cette période, qui se place de plus en plus souvent en août.

Impacts of climate change such as those related to global warming, are observed in many vineyards in France and as in other vineyards in the world. In Burgundy, since 20 years, this evolution of the temperatures changes the maturation conditions, key time for "typicité" and signature of "terroir-millésime". After a study of the changes in Burgundy temperatures (which shows a thermal rupture in 1987-1988), a comparative analysis of the phenologic consequences is performed using thermal data and development dates for two vineyard sites in Côte and HautesCôtes de Beaune (Savigny-lès-Beaune et La Rochepot). The results show a latitudinal and vertical transfer of the temperatures and vine's indexes over a 20-30 year period as well as a double effect of climate warming in the maturation stage attributed to the increase in temperatures and the time shift of this period (which occurs most often in August).

\section{INDEX}

Mots-clés : Bourgogne, changement climatique, Côte et Hautes-Côtes de Beaune, phénologie, vignoble

Keywords : Burgundy, climate change, Côte and Hautes-Côtes de Beaune, phenology, vineyard

\section{AUTEURS}

\section{MALIKA MADELIN}

Malika Madelin est climatologue, Maître de conférences en géographie à l'université ParisDiderot et membre de l'UMR PRODIG. Elle s'intéresse aux échelles fines et en particulier aux relations vigne-climat. Précédemment en poste à l'université de Bourgogne et à l'UMR Centre de Recherches de Climatologie, elle y a effectué des recherches sur le réchauffement climatique dans les vignobles bourguignons. malika.madelin@univ-paris-diderot.fr

\section{BENJAMIN BOIS}

Actuellement Maître de Conférences à l'université de Bourgogne (Institut Universitaire de la Vigne et du Vin) et à l'UMR Centre de Recherches de Climatologie, Benjamin Bois a pour thème de recherche l'agroclimatologie viticole.benjamin.bois@u-bourgogne.fr

\section{JEAN-PIERRE CHABIN}

Maître de Conférences en géographie à l'université de Bourgogne et à l'UMR Centre de Recherches de Climatologie, Jean-Pierre Chabin, actuellement à la retraite, a fortement amorcé et contribué aux travaux de climatologie sur le vignoble de Bourgogne. jeanpierre.chabin@wanadoo.fr 